\title{
Improvements of INCL4 in view of more reliable predictions for RIB facilities
}

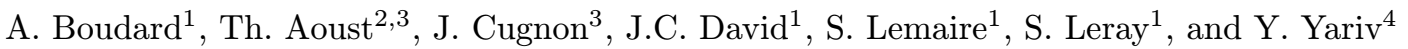 \\ 1 SPhN, CEA-Saclay, 91191 Gif-sur-Yvette Cedex, France \\ 2 SUK-CEN, Boeretang 200, $2400 \mathrm{Mol}$, Belgium \\ 3 University of Liège, Allée du 6 août 17, Bâtiment B5, 4000 Liège 1, Belgium \\ 4 SPhN, CEA-Saclay, 91191 Gif-sur-Yvette Cedex, France, on leave from Soreq NRC, Yavne 81800, Israel
}

Received: January 31, 2007

\begin{abstract}
Some improvements of the Intra-Nuclear Cascade code INCL4 for low energy projectiles are presented and discussed.
\end{abstract}

PACS. 25.40.Sc Spallation reactions

The recent intensive activities in production of Rare Isotope Beams and High Current Spallation Sources led to a revival of interest in reliable predictive modeling of collisions of hadrons with nuclei in the energy range of few tens $\mathrm{MeV}$ to a few $\mathrm{GeV}$. Owing to the complexity of the quantum-mechanical many-body problems, there is a need to develop tractable theories, using suitable approximations for design of new experiments and identification of interesting new phenomena. Such computational tools, implemented into transport codes (e.g. MCNPX, GEANT4) are also indispensable for characterization of the backgrounds in experimental setups and for the radioprotection evaluations.

Good candidates for such modeling are Monte Carlo implementations of Intra-Nuclear Cascade (INC) models (e.g. INCL4 [1], ISABEL [2]) followed by de-excitation models (e.g. ABLA [3], GEM [4]). They reproduce successfully a wide variety of experimental data of hadron-nucleus reactions and they have a strong predictive power, since they use only a small number of parameters, most with clear physical meaning.

They have been successfully implemented into the MCNPX transport code. Considering a clusterisation of nucleons at the surface of the nucleus, INCL4 [5] has also given promising results for production of light particles (up to ${ }^{4} \mathrm{He}$ ) for beam energies above $\sim 500 \mathrm{MeV}$.

The INC models treat the interaction of incoming hadron with the nucleus as a series of independent collisions using free particle-nucleon cross sections and therefore should be valid only above $150-200 \mathrm{MeV}$ and for relatively heavy nuclei.

It was actually found that INCL4, with some refinements [6], may be successfully used well outside this domain. In the region of a few tens of $\mathrm{MeV}$ the calculation is very sensitive to the detailed modeling of the Pauli exclusion principle. In the original INCL4 code, soft nucleon-nucleon collisions are not considered. This is based on the fact that most of them build the mean nuclear potential which is explicitely considered in the model and on the practical fact that they will be rejected after the calculation of the NN collision by the Pauli principle applied on the two outgoing nucleons. In practice, this is done by a cut on the relativistic invariant energy available in the collision. Above $\sim 100 \mathrm{MeV}$ it was checked that the results are insensitive to reasonable changes of this cut contrary to the computation time. In a developing version of INCL4 (named INCL4.3.1), we have refined this cut and actually replaced the cut in energy by a rejection of collisions if both nucleons are below the Fermi level. With the realistic parametrisation of the $\mathrm{NN}$ interaction and the "statistical Pauli Principle" [1] already used in INCL4, this prescription gives very good results for the computed reaction cross-section down to $\sim 10 \mathrm{MeV}$ (figure 1) without increasing the computation time and without changing the results already obtained at high energy.

At such low energy it is however crucial to consider also the deviation of the projectile trajectory due to the Coulomb field of the nucleus. For a proton, this results in a larger impact parameter on the nuclear potential than the value at infinite distance. On the total reaction cross section, the correction can be derived in classical mechanics:

$$
\sigma_{R}=\sigma_{R}^{\prime}\left(1-\frac{V_{c}}{E_{i n}}\right)
$$

with $\sigma_{R}^{\prime}$ the purely nuclear value from INCL (dashed line in figure 1), $V_{c}$ the coulomb potential and $E_{i n}$ the projectile kinetic energy. The good results compared to experimental date in figure 1 means that we can trust the theoretical normalization and this adds confidence in the model down to low energies. The rather large underprediction in the case of a light nuclei as Beryllium could be expected in view of the cluster structure of this nucleus. 

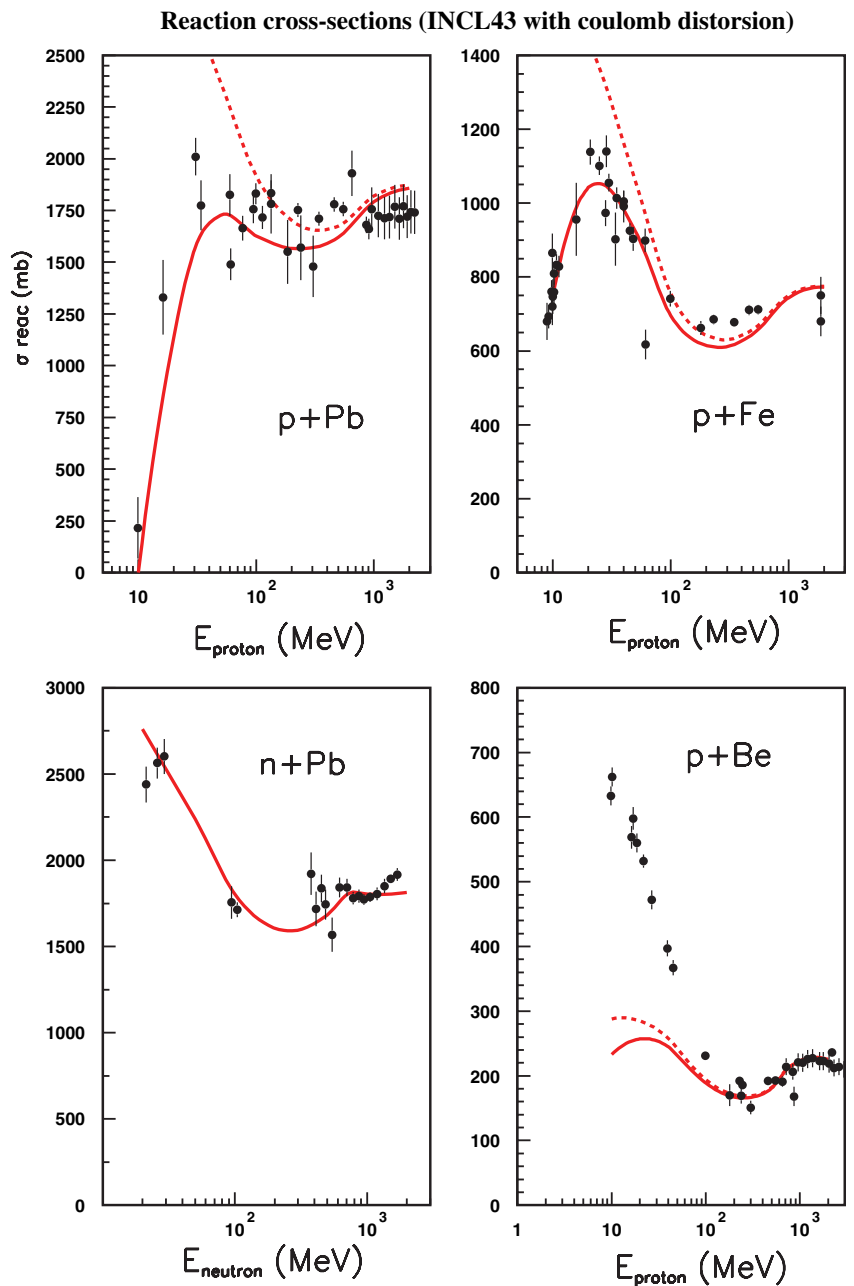

Fig. 1. Reaction cross sections for proton on Lead, Iron and Beryllium and for neutron on Lead as a function of the beam energy. The continuous line is the INCL4.3.1 calculation (dashed line without coulomb correction) compared to date points from compilations [7].

A large amount of data on light particle production at low energy is now available. Among them we present here energy distribution of proton, deuteron, triton and ${ }^{4} \mathrm{He}$ produced by neutrons of $96 \mathrm{MeV}$ on a lead target (figure 2) Compare to the original version of the code ([5]), the geometry of clusterization is more rigorous, the priority between triton and ${ }^{3} \mathrm{He}$ formation (if both possible) is randomly chosen with a 0.5 probability, and the coulomb barriers computed at the nucleus radius plus the r.m.s of the cluster are more realistic. Note that there is no new ad hoc parameters adjusted, only a phase space value to define a cluster and distance from the nucleus to define the clusterization "at the surface" with the same values already used in the $\mathrm{GeV}$ domain. The coupling with the de-excitation code GEM which evaporates all type of light nuclei gives rather promising results (figure 2). Angular distributions (double differential cross-sections not shown here) are of similar quality with a realistic angular dependence for each type of particles.
n+Pb 96MeV, INCL43-GEM (Data from V. Blideanu et al.)
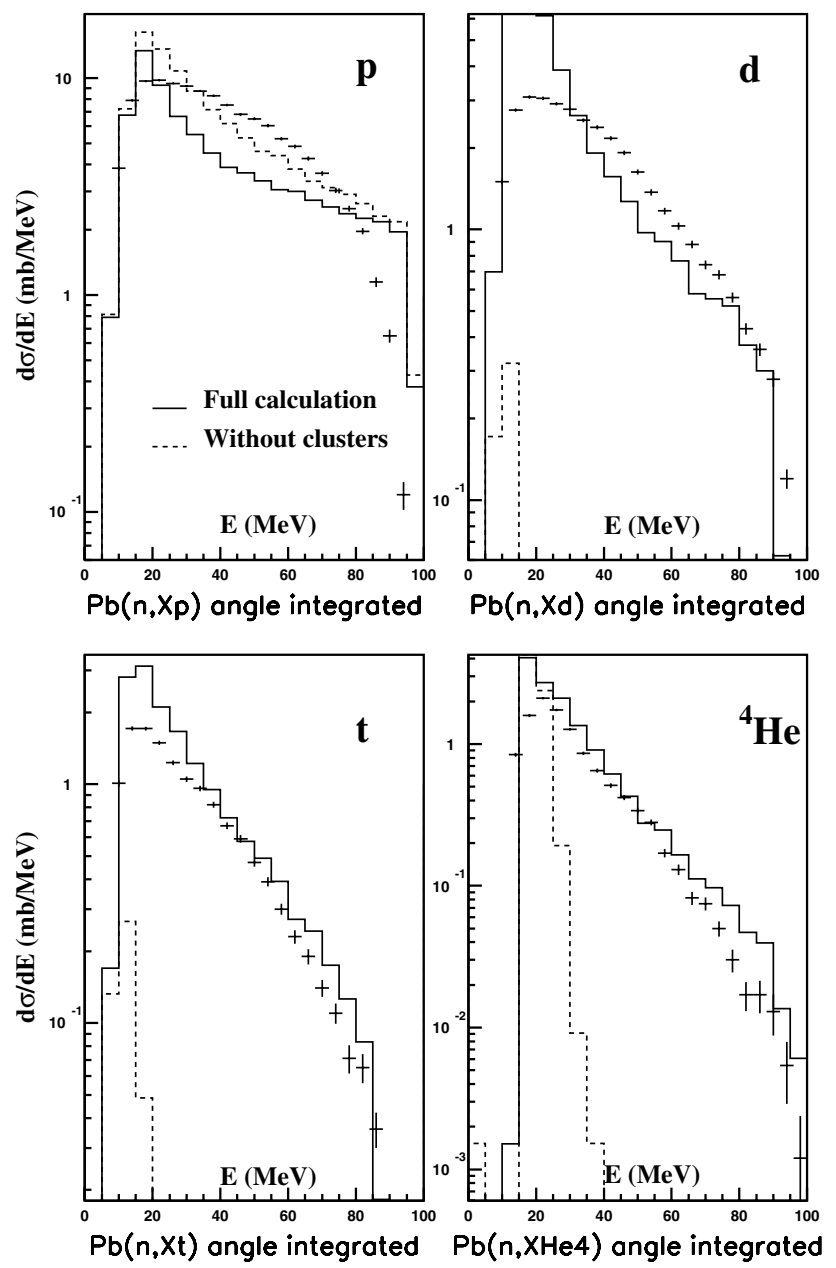

Fig. 2. Energy distribution of $p, d, t$ and ${ }^{4} \mathrm{He}$ produced by neutrons of $96 \mathrm{MeV}$ on a lead target. The continuous line is the INCL4.3.1 calculation coupled with the de-excitation code GEM [4]. The dashed line is the same calculation without production of composite particles in the cascade. The date points are from [8].

The main drawbacks are a substantial lost of proton production and a very large production of low energy deuterons (below $25 \mathrm{MeV}$ ). The evaporation of deuterons being rather small, the observed over prediction is really due to the cascade. A dissociation of the produced deuterons in the queue of the nucleus density evaluated with realistic values will not be enough to correct it. At lower energies ( $\mathrm{n}$ on $\mathrm{Bi}$ at $63 \mathrm{MeV}$ and $41 \mathrm{Mev}$ [9]), the proton and the He production is of similar rather good quality whereas the tritons, but especially the deuterons are more over predicted by the model as the energy goes down especially very close to the Coulomb barrier.

A next step will be to merge these improvements with the sophistications introduced in the nuclear potential [6].

The new INCL version will improve and extend the predictive power of the transport code and make it useable also for lower energy facilities as EURISOL and SPIRAL2. 
A stand-alone version of INCL4-ABLA is available on request (alain.boudard@cea.fr).

\section{References}

1. A. Boudard, J. Cugnon, S. Leray, C. Volant, Phys. Rev. C 66, 044615 (2002)

2. Y. Yariv, Z. Fraenkel, Phys. Rev. C 24, 448 (1981)
3. A.R. Junghans et al., Nucl. Phys. A 629, 635 (1998)

4. S. Furihata, T. Nakamura, J. Nucl. Sci. Technol. 2, 720 (2002)

5. A. Boudard, J. Cugnon, S. Leray, C. Volant, Nucl. Phys. A 740, 195 (2004)

6. Th. Aoust, J. Cugnon, Eur. Phys. J. A 21, 79 (2004)

7. R.E. Prael, M.B Chadwick, Los Alamos National Laboratory preprint LA-UR-97-1744, 1997

8. V. Blideanu et al., Phys. Rev C 70, 014607 (2004)

9. E. Raeymackers et al., Nucl. Phys. A 726, 210 (2003) 